\title{
Effectiveness of a minimal resource fracture liaison service
}

\author{
K. F. Axelsson ${ }^{1,4,5} \cdot$ R. Jacobsson ${ }^{2}$ D. Lund ${ }^{3}$ - M. Lorentzon ${ }^{4,5}$
}

Received: 26 February 2016 / Accepted: 13 May 2016/Published online: 26 May 2016

(C) The Author(s) 2016. This article is published with open access at Springerlink.com

\begin{abstract}
Purpose The purpose of this study was to investigate if a 2year intervention with a minimal resource fracture liaison service (FLS) was associated with increased investigation and medical treatment and if treatment was related to reduced refracture risk

Methods The FLS started in 2013 using existing secretaries (without an FLS coordinator) at the emergency department and orthopaedic wards to identify risk patients. All patients older than 50 years of age with a fractured hip, vertebra, shoulder, wrist or pelvis were followed during 2013-2014 $(n=2713)$ and compared with their historic counterparts in 2011-2012 $(n=2616)$ at the same hospital. Re-fractures were $\mathrm{X}$-ray verified. A time-dependent adjusted (for age, sex, previous fracture, index fracture type, prevalent treatment, comorbidity and secondary osteoporosis) Cox model was used. Results The minimal resource FLS increased the proportion of DXA-investigated patients after fracture from 7.6 to $39.6 \%$ $(p<0.001)$ and the treatment rate after fracture from 12.6 to $31.8 \%$, which is well in line with FLS types using the
\end{abstract}

M. Lorentzon

mattias.lorentzon@medic.gu.se

1 Department of Orthopaedic Surgery, Skaraborg Hospital, Skövde, Sweden

2 Sahlgrenska Academy, Gothenburg University, Gothenburg, Sweden

3 School of Bioscience, University of Skövde, Skövde, Sweden

4 Geriatric Medicine, Institute of Medicine, University of Gothenburg, Sahlgrenska University Hospital, Building K, 6th Floor, Mölndal 431 80, Sweden

5 Center for Bone Research at the Sahlgrenska Academy, Institute of Medicine, University of Gothenburg, Gothenburg, Sweden conventional coordinator model. Treated patients had a $51 \%$ lower risk of any re-fracture than untreated patients (HR 0.49, $95 \%$ CI $0.37-0.65 p<0.001$ ).

Conclusions We found that our minimal resource FLS was effective in increasing investigation and treatment, in line with conventional coordinator-based services, and that treated patients had a $51 \%$ reduced risk of new fractures, indicating that also non-coordinator based fracture liaison services can improve secondary prevention of fractures.

Keywords Efficiency $\cdot$ FLS $\cdot$ Fracture $\cdot$ Fracture liaison service $\cdot$ Osteoporosis

\section{Introduction}

In Sweden, the fourth most important cause of illness is fallrelated injuries [1]. Falls often cause fractures, and the yearly fracture-related cost of osteoporosis in Sweden has been estimated to be $€ 0.5$ billion [2]. Fractures are common and dangerous. At the age of 50, the risk of sustaining a fragility fracture (i.e. a fracture caused by an injury that would be insufficient to fracture a normal bone) during the remaining lifetime is $50 \%$ for women and $20 \%$ for men [3]. Fragility fractures are associated with an increased morbidity and mortality, especially hip and vertebral fractures in which the risk of mortality is twice as high for patients older than 80 years compared to age-matched controls [4].

There is solid evidence that medical treatment of osteoporosis reduces fracture rate in postmenopausal women, especially with low BMD and high fracture risk [5]. Alendronate treatment leads to considerable relative risk reduction (RRR), $45 \%$ for vertebral fractures and $40 \%$ for hip fractures [6]. With a yearly dose of zoledronic acid, the RRR is even higher, 
$70 \%$ for vertebral fractures and $41 \%$ for hip fractures [7]. Denosumab treatment has also proven effective to reduce fracture risk. For women older than 75 years or with T-score $<2.5$ SD, the RRR for a hip fracture was $61 \%$, and for patients with multiple vertebral fractures, the RRR for subsequent vertebral fracture was $55 \%$ [8].

A patient with a prior fracture has an increased risk of a new fracture [9]. For most fracture sites, the risk is doubled, but a patient with a vertebral fracture has a 4-fold increased risk of a new vertebral fracture [9]. In spite of welldocumented evidence for fracture preventing medication, only a minority receive medical treatment within 6-12 months after an osteoporotic fracture (14\% of Swedish women and $3 \%$ of men, aged 50 years or more) [10].

In an effort to increase the proportion of treated patients after a fracture, structured prevention programs (fracture liaison services, FLS) have been introduced to identify, investigate and reduce patients at risk of new fractures. Demonstrably, FLS programs show an increase in the number of patients investigated and treated [11, 12].

However, there are few studies attempting to report on the FLS main objective, which is prevention of new fractures. Furthermore, previously reported studies may raise quality concerns [13-17]. Two studies created a control group from the patients who chose not to participate in the FLS $[13,15]$. Two other studies used another hospital without an FLS as a control $[16,17]$. Both approaches raise selection bias issues. Three of the studies used questionnaires to record re-fractures [13-15]. However, self-reported fractures have been associated with 11-29\% false positives [18-20] and $7 \%$ false negatives [19]. Thus, using this method to record fractures introduces an error of the same magnitude as the expected effect from treatment [6]. The previously reported studies only included between 41 and 225 re-fractures.

The gold standard when introducing an FLS is to use a coordinator to select patients and manage the patient flow [21]. However, this would require specific additional funds that may not be available at all hospitals. Therefore, we investigated an FLS without a coordinator, introduced with minimal organisational changes. The aim of the performed study was to investigate if a minimal effort FLS (2-year intervention) was associated with increased investigation and medical treatment in a large cohort $(n=5,329)$ of men and women with fracture.

\section{Materials and methods}

\section{Fracture liaison service}

Skaraborg Hospital, a Swedish regional hospital covering 265,000 inhabitants, started an FLS on 1 January 2013 and included all patients older than 50 years seeking care for an osteoporotic fracture (defined as a fracture of the wrist, upper arm, hip, vertebra or pelvis). The FLS comprised four steps.

1. Risk patient identification: A total of $\sim 30$ secretaries at the emergency department and the orthopaedic clinic were responsible for identifying risk patients and order a bone density measurement (DXA) and fracture risk (FRAX) assessment based on ICD-10 fracture codes in the patient charts. Patients with pathological fractures or who were deceased prior to DXA referral were excluded.

2. DXA examination and FRAX assessment: Patients were invited to the hospital's DXA unit where experienced nurses performed the DXA examination as well as a FRAX assessment.

3. DXA and FRAX evaluation: Based on regional and national guidelines [22], an experienced endocrinology consultant assessed the bone densitometry results and clinical risk factors, defined a diagnosis and provided treatment recommendation and follow-up advice, which were sent to the patient's primary care physician.

4. Treatment initiation: Based on the recommendation and in consultation with the patient, the primary care physician initiated treatment in order to reduce the risk for a new fracture. The primary care physician was responsible to rule out secondary osteoporosis and choose a suitable type of osteoporosis medication.

Our FLS model had two key elements: the delegation of referral power to secretaries and the detailed consultant response, the former to enable organisational stability and robustness and the latter to reduce the workload for the receiving doctor.

During the first year, 2013, patients' relatives or staff at nursing homes often cancelled the examination due to perceived high patient age or morbidity. This leads to a relatively high DXA cancellation rate among the elderly, $50 \%$ for patients over 80 years compared to $23 \%$ for $50-79$ years $(p<0.001)$. By cancelling the DXA, the patient also lost the possibility of being eligible for treatment through the FLS. Therefore, starting January 2014, the hospital decided to offer treatment with a yearly intravenous infusion of zoledronic acid $(5 \mathrm{mg})$ to all patients older than 80 years with a hip fracture, without prior DXA examination.

\section{Study design}

The potential FLS effect was investigated comparing the intervention period, 2013-2014, with the control period, 20112012. All patients older than 50 years with an index osteoporotic fracture, the types targeted by the FLS, were included in the study. Patients with the selected fracture types represented $56 \%$ of all fractured patients. The intervention group consisted of patients presenting with an osteoporotic fracture 
during 2013-2014 $(n=2616)$, i.e. when the FLS had started. The control group consisted of historical controls 2011-2012 $(n=2713)$. The follow-up time varied from 0 to 2 years, i.e. each period was truncated at 31 December 2012 and 2014 respectively in order to achieve group symmetry and to avoid crossover effects. In order to assess potential fracture risk reduction from medical treatment, all treated patients, regardless of period, were compared with the non-treated patients. Subgroup analysis was performed on hip fracture patients above 80 years of age since they were handled slightly differently in the process.

\section{Definition of variables}

In this study, the term fracture refers to a fracture event, i.e. one or more fractures at the same occasion, with an ICD-10 diagnosis that starts with S12, S22, S32, S42, S52, S62, S72, $\mathrm{S} 82, \mathrm{~S} 92, \mathrm{~T} 02, \mathrm{~T} 08, \mathrm{~T} 10, \mathrm{~T} 12, \mathrm{~T} 142$ or M485. If a secretary sent a referral for DXA as instructed, it was automatically registered in the digitalized patient record. The age was set at the time of the index fracture, and gender was derived from the patient's social security number.

The follow-up time in the study was calculated from the fracture date (or if the patient was admitted, from the date of discharge) to the actual event, where event refers to DXA scan, medical treatment, a subsequent fracture or death, depending on analysed outcome. The follow-up time was truncated if a patient moved from the region, died or if the followup period ended.

Previous fracture was defined as any other fracture before the date of the index fracture, stretching exactly 14 years back in time.

Three types of prevalent medications were accounted for: (i) active medical treatment for osteoporosis, (ii) calcium and/ or vitamin D and (iii) glucocorticoids. Prevalent active medical treatment for osteoporosis was included as number of days during the year prior to fracture with bisphosphonates, denosumab, strontium ranelate or hormonal treatment (p.o. or transdermal). Similarly, a separate variable was calculated for calcium and/or vitamin D. Prevalent glucocorticoid treatment was defined as milligrams of prednisolone equivalents during the year prior to fracture. The Prednisolone equivalents were calculated using conversion factors: betamethasone (8.33), dexamethasone (6.67), methylprednisolone (1.25), hydrocortisone $(0.25)$ and cortisone $(0.2)$.

Incident medical treatment was defined as number of days of treatment, which started at the first date of medication retrieval from the pharmacy after the index fracture and ended when prescription ended, a subsequent fracture or death occurred or if the patient moved out of the area. For prescription medication, treatment refers to the patient retrieving medication at the pharmacy whereas parenteral treatment administered at the hospital was registered in the patient's record.
Categorical variables were created for prevalent illnesses, if the ICD-10 diagnosis was present in the hospital records within 10 years prior to index fracture. The illnesses included osteoporosis with or without fracture, rheumatoid arthritis and all diagnoses with prevalence higher than $5 \%$ that might correlate with fracture risk. We also created a variable for secondary osteoporosis according to FRAX and all its components (insulin-dependent diabetes, hyperthyroidism, hypogonadism, malnutrition, osteogenesis imperfecta or chronic liver disease). A modified Charlson comorbidity index was used to summarize and quantify comorbidity. The index was calculated as a sum of the following diseases, ICD-10 codes in parenthesis: one point each for an ischaemic heart disease (I20-I25); congestive heart failure (I50); cerebrovascular disease (I60-I69); disease of arteries, arterioles and capillaries (I70-I79); diabetes (E10-14); dementia (F00-F03); chronic pulmonary disease (J43-J46); and chronic liver disease (parts of K70-K77); two points each for renal failure (N17-N19), hemiplegia (G81); lymphoma or leukemia (C81-C96); and tumor without metastasis (C00-C76, C80, C97, 5-year history); and six points for metastatic solid tumor (C77-C79) - 10-year history if not stated otherwise.

\section{Statistics}

Chi-square tests were used on categorical variables in order to investigate statistical differences between the control and intervention groups in terms of comorbidity, previous medications and known risk factors. Differences between the continuous variables were investigated using $t$ test. A Cox proportional hazard regression model was used for time-dependent analysis. When DXA or treatment was the outcome, adjustment was made only for age and gender (crude). When analysing risk of new fracture or death, adjustment was also made for previous fracture, index fracture type (hip fracture, vertebral fracture or other), prevalent medical treatment, prevalent calcium or vitamin $\mathrm{D}$ treatment, prevalent glucocorticoid treatment, prevalent osteoporosis diagnosis, rheumatoid arthritis and secondary osteoporosis according to FRAX as well as a modified Charlson morbidity index (fully adjusted). A $p$ value less than 0.05 was considered significant.

\section{Statistical power calculation}

The primary aim was to study the FLS effect on DXA utilization and treatment rates. Assuming 1500 osteoporotic fractures occur in Skaraborg per year, the mean follow-up time in this 2-year study is 1 year (different starting points, fixed end-point). Approximately $14 \%$ receive treatment after fracture [10]. Assuming an alpha-value of 0.05 , any postintervention treatment rate above $16 \%$ would render a power above $80 \%$. Since DXA measurements in most cases precede treatment, we expect to find similar or larger increases in DXA 
utilization and therefore increased statistical power for this end-point.

Regarding re-fractures in approximately 3000 patients in each group, mean follow-up time of 1 year, assuming an alpha-value of 0.05 , an annual $8 \%$ re-fracture rate without treatment, an increase from 14 to $50 \%$ in treatment rates and a $50 \%$ reduction of re-fractures from treatment would render a statistical power of $82 \%$. However, assuming a 4month average delay from index fracture to start of treatment, the statistical power falls to $51 \%$ for the re-fracture end-point.

\section{Assessment of BMD}

Assessment of BMD (aBMD, g/ $\mathrm{cm}^{2}$ ) was measured at the hip, femoral neck and lumbar spine (L1-L4) using a Lunar Prodigy (GE Healthcare). The same DXA machine was used for all measurements. The coefficients of variations (CV) ranged from 0.7 to $1.5 \%$.

\section{Information sources}

The hospital's digitalized patient records were used to identify patients with fractures during 2011-2014. In order to differ records of re-visits regarding an old fracture from records of new fractures, manual verification was performed. If a patient had fracture entries on more than one occasion, that patient's hospital record was reviewed and only entries representing true fracture occasions were kept. Thus, all re-fractures were manually verified in the patient's hospital records.

Information on the identified cohort concerning previous fractures, current and previous diseases and medication as well as date of DXA examination was also collected from the hospital's patient records. Date of death and moving in or out of the hospital's district was collected from the Swedish Population Registry.

\section{Results}

\section{Patient characteristics}

A total of 5329 patients were included in the study, with 2616 in the intervention group and 2713 in the control group. The accumulated follow-up time was 5014 years, with 2481 years in the intervention group and 2534 years in the control group. The mean follow-up time (mean \pm SD) was 344 days (346 \pm 219 days in the intervention versus $341 \pm 221$ days in the control group, $p=0.39$ ).

\section{Baseline characteristics}

Baseline characteristics are presented in Table 1. Apart for slightly less patients with hypertension in the intervention group, there were no significant differences between the intervention group and the control group with regard to sex, age, proportion of previous fracture, prevalence of osteoporosis, rheumatoid arthritis, secondary osteoporosis according to FRAX, modified Charlson comorbidity index, other possible fall- or fracture-related diseases with prevalence above $5 \%$ or type of index fracture (Table 1).

In the subgroup hip fracture patients above 80 years, the intervention group was slightly less inclined to have previous glucocorticoid treatment, but otherwise no significant differences were observed (Table 1).

In the group selected for treatment, the prevalence of previous fracture was higher, as well as the proportion of women and vertebral index fracture, than in the untreated group (Table 1). Patients selected for treatment were slightly healthier according to Charlson (Table 1) and less prone to have dementia $(2.1$ versus $4.0 \%, p<0.01)$ than their untreated counterparts were. Also, patients selected for treatment were more inclined to already have treatment for osteoporosis as well as glucocorticoid treatment (Table 1).

\section{FLS patient identification}

Of all the osteoporotic fractures in 2013-2014, $11 \%$ were omitted by the system, i.e. occurring in a unit with no FLS secretary. Of the patients treated at an FLS unit, the secretaries identified $75 \%$ of the defined osteoporotic fractures. Admitted patients had a higher likelihood of being included than did patients visiting the emergency room (88 versus $61 \%, p<0.001)$. The likelihood of being included also differed by index fracture type: hip $92 \%$, vertebral $60 \%$, wrist $76 \%$, shoulder $55 \%$ and pelvic $67 \%(p<0.001)$.

\section{Increased likelihood of DXA examination}

The proportion of patients being examined with DXA after a fracture increased from $7.6 \%$ in the control period to $39.6 \%$ in the intervention period $(p<0.001)$. The time to DXA examination (mean $\pm \mathrm{SD}$ ) after index fracture decreased from $133 \pm 119$ to $85 \pm 67$ days ( $p<0.001$ ), and the patients examined had no statistical difference in terms of femoral neck $\operatorname{BMD}(0.72 \pm 0.13$ versus $0.73 \pm 0.13, p=0.36)$. Age- and sex-adjusted time-dependent Cox regression analysis of the intervention period versus the control period showed a 7fold increased likelihood of being examined with a DXA (HR 7.0, 95\% CI 6.0-8.1 $p<0.001$ ) in the intervention period (Fig. 1a). These results were highly similar when fully adjusted (HR 7.1, $95 \%$ CI 6.1-8.2, $p<0.001)$.

\section{Increased likelihood of medical treatment}

There was no significant difference in prevalent osteoporosis treatment rates between the control $(9.2 \%)$ and the 


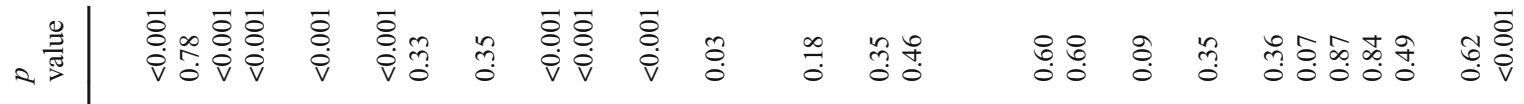
吾突

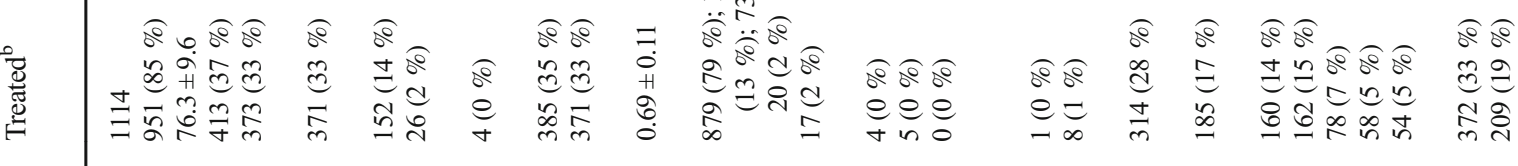

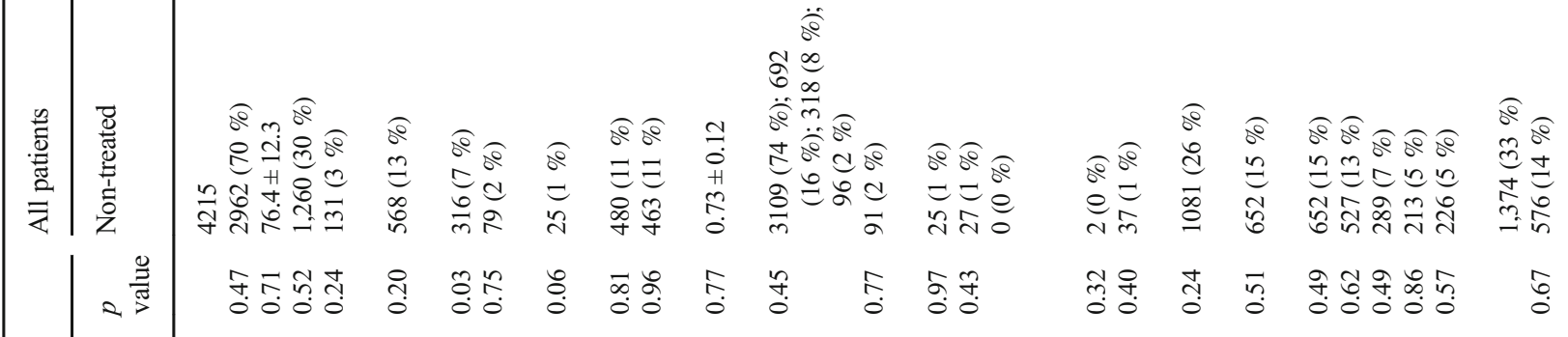

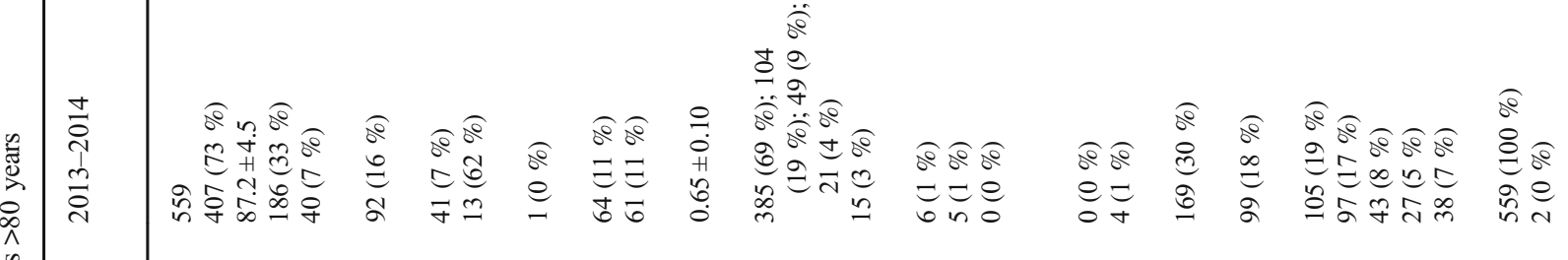

के

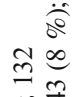

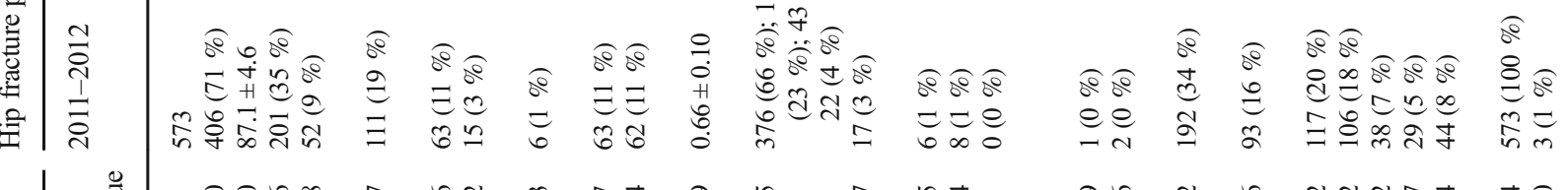

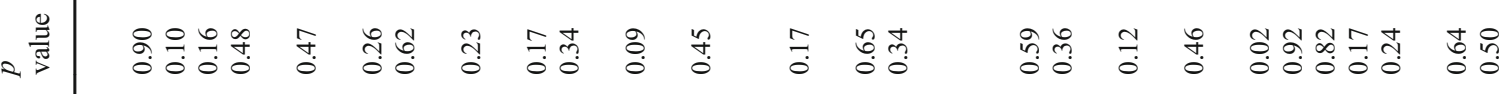

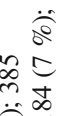

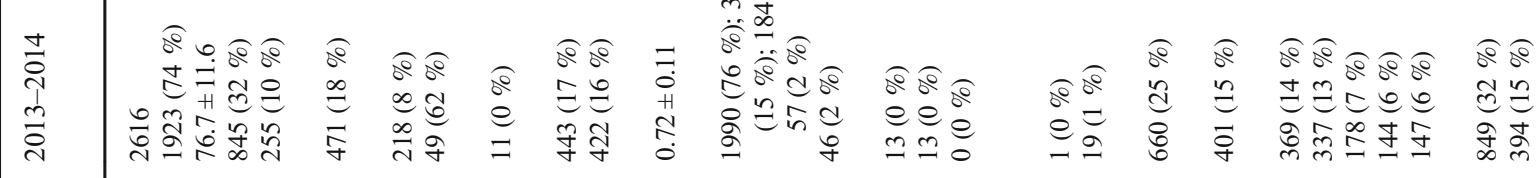

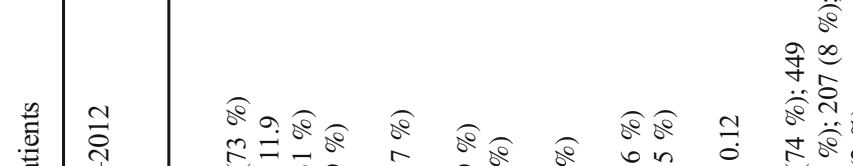

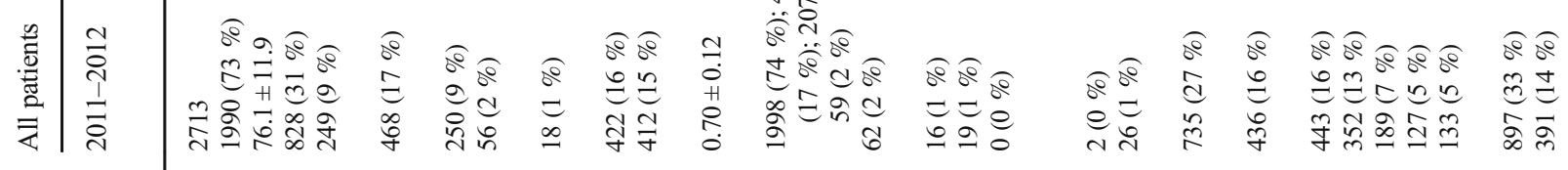


intervention period $(9.7 \%)$. While the treatment rate after a fracture only increased to $12.6 \%(n=341)$ in the control period, it increased to $31.8 \%(n=833)$ in the intervention period $(p<0.001)$. Excluding patients with previous treatment, there was no significant difference in time to treatment (mean \pm SD) in the control period ( $123 \pm 115$ days) compared to the intervention period (118 \pm 79$), p=0.33$. Age- and sex-adjusted time-dependent Cox regression analysis of the intervention period versus the control period revealed a 2.8 -fold increased likelihood of being treated (HR 2.8, $95 \%$ CI 2.5-3.2, $p<0.001$, Fig. 1b). These results were highly similar when fully adjusted (HR 2.6, $95 \%$ CI 2.3-2.9, $p<0.001$ ). A breakdown of types of treatment showed that the proportion of zoledronic acid and denosumab increased dramatically in the intervention group (Table 2).

\section{Re-fractures}

There were a total of 469 new fractures in 444 patients, and the most common re-fracture site was the hip (Table 3 ). There was no significant difference in re-fracture rate in the control period compared to the intervention period-228 (8.4\%) versus $216(8.3 \%), p=0.85$. Time to new fracture (mean \pm SD) was $207 \pm 168$ days in the control and $200 \pm 163$ in the intervention period, $p=0.66$. Using a time-dependent Cox analysis, the risk reduction was also non-significant (crude HR 0.95, $95 \%$ CI $0.79-1.14, p=0.59$, and fully adjusted HR 0.95, $95 \%$ CI $0.79-1.14, p=0.60)$.

\section{Deaths}

There was no significant difference in mortality rate in the control period compared to the intervention period: 361 (13.3\%) versus $320(12.2 \%), p=0.24$. Time to death (mean $\pm \mathrm{SD}$ ) was $178 \pm 169$ days in the control and $186 \pm 162$ in the intervention period, $p=0.56$. A time-dependent Cox analysis showed that the mortality in the intervention group tended to be lower, but the reduction was not statistically significant (crude HR 0.87, $95 \%$ CI $0.75-1.01, p=0.07$, and fully adjusted HR 0.88, $95 \%$ CI 0.76-1.03, $p=0.11$ ).

\section{Subgroup analysis}

In hip fracture patients older than 80 years, the ratio of DXA measurement after index fracture increased from 1.0 to $26.1 \%$ or using a Cox proportional hazard analysis adjusted for age and sex, which is a 32-fold increased likelihood (HR 32.4, $95 \%$ CI 14.3-73.3, $p<0.001)$.

The chance of receiving medical treatment increased from 8.4 to $28.3 \%$, or using Cox proportional hazard analysis adjusted for age and sex, which is a 3.9-fold increased likelihood (HR 3.9, $95 \%$ CI 2.8-5.4, $p<0.001$ ). Just like for the whole group, when excluding patients with previous treatment, there 
Fig. 1 Follow-up after index fracture. Periods 2011-2012 (no FLS) versus 2013-2014 (FLS). a DXA examination, $\mathbf{b}$ pharmacological treatment. Adjusted for age and sex
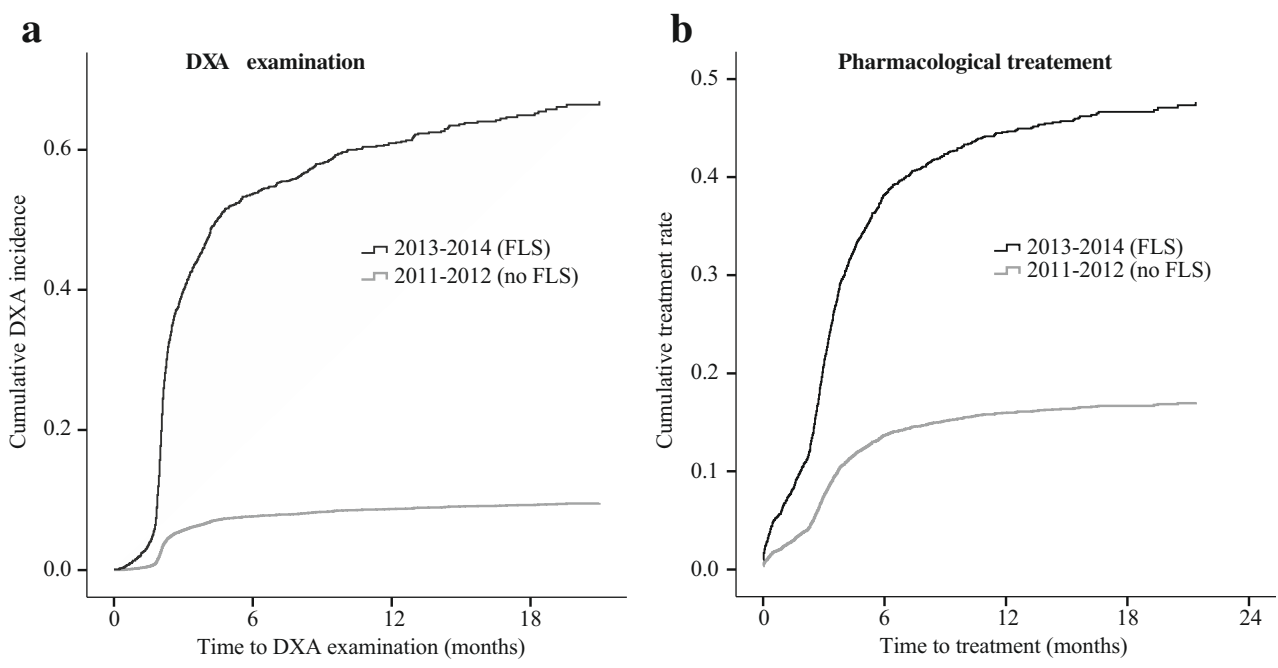

was no difference in time to treatment (mean $\pm \mathrm{SD}$ ), 127 \pm 119 days in the control versus $105 \pm 47$ days in the intervention group, $p=0.24$.

Regarding re-fractures, there was no significant difference in time to new fracture, $174 \pm 168$ days versus $160 \pm 152$ days $(p=0.66)$, but a significant reduction in re-fracture rate from 11.9 to $7.8 \%(p=0.02)$ was observed comparing the control and FLS periods. Using the time-dependent Cox model adjusted for age and sex, the FLS period was associated with a $35 \%$ reduction of re-fracture rate (HR $0.65,95 \%$ CI $0.45-$ $0.95, p=0.03$ ). This reduction was similar (HR $0.6595 \% \mathrm{CI}$ $0.44-0-94, p=0.02$ ) when fully adjusted.

\section{Treated patients had reduced risk of re-fracture}

Regardless of period, the treated patients had a significantly lower fracture rate versus the non-treated patients (6.6 versus $8.8 \%, p=0.02$ ) as well as longer mean time to new fracture,
294 days versus 185 days $(p<0.001)$. Using a time-dependent Cox model, treated patients had a $40 \%$ reduction of risk for new fracture (crude HR $0.60,95 \%$ CI $0.47-0.77, p<0.001$ ) or $51 \%$ after adjustments for covariates (HR 0.49, $95 \%$ CI $0.37-0.65, p<0.001$, Fig. 2) than did untreated patients.

Using a fully adjusted Cox analysis, the risk reduction of specific future fractures was even higher: for hip fractures $64 \%(p<0.01)$, for vertebral fracture $73 \%(p<0.01)$, for major osteoporotic fractures $67 \%(p<0.001)$ and for patients with multiple future fracture events $63 \%(p=0.047)$. In the subgroup of patients older than 80 years, the risk reduction was slightly higher than for the treated group as a whole, $54 \%$ $(p<0.001)$ (Table 4).

\section{Mortality}

The association between the Charlson comorbidity index and mortality was analysed using a time-dependent Cox

Table 2 Pharmacological treatment

\begin{tabular}{|c|c|c|c|c|}
\hline & \multicolumn{2}{|l|}{ Before $^{\mathrm{a}}$ index fracture } & \multicolumn{2}{|l|}{ After ${ }^{\mathrm{b}}$ index fracture } \\
\hline & 2011-2012 (no FLS) & 2013-2014 (FLS) & 2011-2012 (no FLS) & 2013-2014 (FLS) \\
\hline $\begin{array}{l}\text { Alendronate w/wo cholecalciferol, } \\
\text { risedronate, chlodronate, } \\
\text { pamidronate, ibandronate }\end{array}$ & $194(7.2 \%)$ & $173(6.6 \%)$ & $281(10.4 \%)$ & $641(24.5 \%)$ \\
\hline Zoledronic acid & $3(0.1 \%)$ & $8(0.3 \%)$ & $3(0.1 \%)$ & $83(3.2 \%)$ \\
\hline Denosumab (Prolia) & $6(0.2 \%)$ & $10(0.4 \%)$ & $8(0.3 \%)$ & $38(1.5 \%)$ \\
\hline All hormonal treatment, excl. vaginals & $46(1.7 \%)$ & $64(2.4 \%)$ & $30(1.1 \%)$ & $27(1 \%)$ \\
\hline Strontium ranelate (Proteolos) & $0(0 \%)$ & $0(0 \%)$ & $1(0 \%)$ & $1(0 \%)$ \\
\hline Teriparatid (Forsteo) & $0(0 \%)$ & $0(0 \%)$ & $0(0 \%)$ & $1(0 \%)$ \\
\hline Total active osteoporotic treatment & $249(9.2 \%)$ & $255(9.7 \%)$ & $323(11.9 \%)$ & $791(30.2 \%)$ \\
\hline No treatment & $2464(90.8 \%)$ & $2361(90.3 \%)$ & $2390(88.1 \%)$ & $1825(69.8 \%)$ \\
\hline Number of patients & 2713 & 2616 & 2713 & 2616 \\
\hline
\end{tabular}

${ }^{\mathrm{a}}$ One year prior to fracture

${ }^{\mathrm{b}}$ After index fracture but before potential re-fracture 
Table 3 Re-fractures

\begin{tabular}{ll}
\hline Re-fracture site & Number (\%) \\
\hline Hip & $133(28 \%)$ \\
Vert & $64(14 \%)$ \\
Wrist & $56(12 \%)$ \\
Pelvic & $54(11 \%)$ \\
Shoulder & $34(7 \%)$ \\
Lowerleg & $31(7 \%)$ \\
Distal femur & $19(4 \%)$ \\
Costal & $16(3 \%)$ \\
Elbow & $13(3 \%)$ \\
Finger & $8(2 \%)$ \\
Hand & $8(2 \%)$ \\
Toe & $7(1 \%)$ \\
Clavicle & $7(1 \%)$ \\
Other & $19(4 \%)$ \\
Number of re-fractures & $469(100 \%)$ \\
Patients with multiple re-fracture sites & 25 \\
Number of patients with re-fractures & 444 \\
\hline
\end{tabular}

regression, adjusted for sex and age with death as outcome. For each increased point in Charlson, the mortality was increased by $30 \%$ (HR 1.30, $95 \%$ CI 1.21-1.39, $p<0.001$ ). The mortality rate was significantly lower in the treated group compared to the untreated group (6.4 versus $14.5 \%$, $p<0.001$ ), and the mean time to death was longer in the treated group compared to the untreated group (244 versus

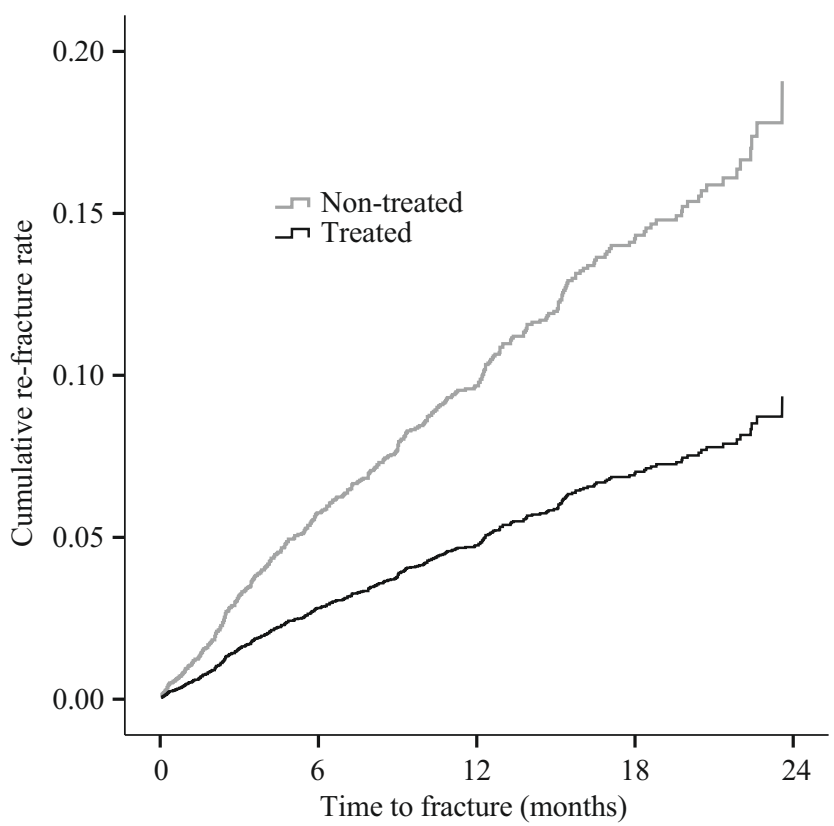

Fig. 2 Cumulative incidence of any re-fracture. Period 2011-2014. Nontreated versus treated. Adjusted for age, sex, previous fracture, previous osteoporosis, previous treatment, Charlson comorbidity index, secondary osteoporosis according to FRAX, rheumatoid arthritis and type of index fracture
175 days, $p<0.01)$. Time-dependent Cox analysis showed that the treated group had a $56 \%$ (crude HR 0.44, $95 \% \mathrm{CI}$ $0.34-0.56, p<0.001$ ) or $66 \%$ (fully adjusted HR $0.34,95 \%$ CI $0.25-0.44, p<0.001)$ lower risk of mortality than did untreated patients.

\section{Discussion}

This study shows a remarkable 7-fold increase in patients receiving DXA examination as well as a 2.8 -fold increased chance of receiving pharmacological osteoporosis treatment as a result of a minimal resource (non-coordinator-based) FLS. Patients receiving osteoporosis medication had a $51 \%$ lower re-fracture rate. With a re-fracture rate of $8.8 \%$ for the untreated, treatment was associated with an absolute risk reduction (ARR) of $4.5 \%$, which translates to 66 prevented fractures per year in our total population sustaining a total of 1332 fractures yearly.

The reduction of re-fracture risk in the intervention group was not significant for the study as a whole, but in the subgroup of hip fracture patients older than 80 years, the FLS was associated with a $35 \%$ significantly lower re-fracture risk. During the second year (2014) the FLS was used, these patients were able to receive zoledronic acid treatment (at the hospital or in their homes) without prior DXA assessment, which increased the proportion receiving treatment even further. FLS patients in this category had a lower proportion of prevalent glucocorticoid treatment, but were similar to controls in regard to comorbidity, prevalent fracture and other medical treatments. Even though the FLS was associated with a reduced re-fracture rate in Cox models adjusted for glucocorticoid use and other covariates, it cannot be ruled out that the selection of patients contributed to the association. A post hoc power analysis based on actual risk reduction from 11.9 to $7.8 \%$ using an alpha-value of $5 \%$ revealed a statistical power of $89 \%$ in this particular subgroup.

The mortality rate among the patients with a re-fracture was 16.6 versus $12.5 \%$ among patients without a re-fracture, indicating a relationship between re-fracture and mortality. However, when comparing the treated patients with the untreated, the reduction in mortality $(66 \%)$ was greater than the reduction in re-fracture $(51 \%)$, even after adjustment for sex, age, comorbidity, previous illnesses and medication. As expected, the Charlson comorbidity index was associated with mortality. However, the large mortality risk reduction in patients selected for medical treatment suggests that treated patients were healthier, although we could not account for this using our available data. In a meta-analysis of randomized controlled trials, osteoporosis treatment has been shown to reduce mortality by $11 \%$ [23].

In our hospital, there is no specific department for osteoporosis patients, which is partly why the distributed secretary- 
Table 4 Time-dependent Cox regression for treated versus non-treated on specific fracture events

\begin{tabular}{|c|c|c|c|c|c|}
\hline Age at index fracture & Follow-up event & Number of re-fractures & Adjustment $^{\mathrm{a}}$ & Risk reduction & HR $95 \%$ CI \\
\hline \multirow[t]{2}{*}{ Above 50} & \multirow[t]{2}{*}{ Any new fracture } & \multirow[t]{2}{*}{444} & Crude & $40 \%$ & $0.60(0.47-0.77)$ \\
\hline & & & Adjusted & $51 \%$ & $0.49(0.37-0.65)$ \\
\hline \multirow[t]{2}{*}{ Above 50} & \multirow{2}{*}{$\begin{array}{l}\text { Any new fracture and thereafter } \\
\text { at least one more fracture event }\end{array}$} & \multirow[t]{2}{*}{47} & Crude & $54 \%$ & $0.46(0.2-1.1)$ \\
\hline & & & Adjusted & $64 \%$ & $0.37(0.14-0.99)$ \\
\hline \multirow[t]{2}{*}{ Above 50} & \multirow[t]{2}{*}{ Major osteoporotic fracture ${ }^{\mathrm{b}}$} & \multirow[t]{2}{*}{279} & Crude & $59 \%$ & $0.41(0.28-0.59)$ \\
\hline & & & Adjusted & $67 \%$ & $0.33(0.22-0.51)$ \\
\hline \multirow[t]{2}{*}{ Above 50} & \multirow[t]{2}{*}{ Vertebral fracture } & \multirow[t]{2}{*}{64} & Crude & $48 \%$ & $0.52(0.25-1.05)$ \\
\hline & & & Adjusted & $73 \%$ & $0.27(0.12-0.61)$ \\
\hline \multirow[t]{2}{*}{ Above 50} & \multirow[t]{2}{*}{ Proximal femur fracture } & \multirow[t]{2}{*}{133} & Crude & $67 \%$ & $0.33(0.18-0.6)$ \\
\hline & & & Adjusted & $64 \%$ & $0.36(0.19-0.68)$ \\
\hline \multirow[t]{2}{*}{ Above 80} & \multirow[t]{2}{*}{ Any new fracture } & \multirow[t]{2}{*}{287} & Crude & $42 \%$ & $0.58(0.42-0.81)$ \\
\hline & & & Adjusted & $54 \%$ & $0.46(0.32-0.67)$ \\
\hline
\end{tabular}

${ }^{\mathrm{a}}$ Crude $=$ adjusted for age and sex. Adjusted = adjusted for age, sex, previous fracture, previous osteoporosis, previous treatment, Charlson comorbidity index, secondary osteoporosis according to FRAX, rheumatoid arthritis and type of index fracture

${ }^{\mathrm{b}}$ Fractured hip, vertebra, wrist or shoulder

based model was chosen instead of a central coordinatorbased model, which would have been more exposed to organisational changes and required additional staff. If all types of fractures would have been included in the FLS, we speculate that the secretaries' ability to recall and identify patients to refer to DXA would increase, which could possibly have reduced the $25 \%$ omitting rate.

To compare our minimal resource FLS with a conventional coordinator-based FLS, the only procedure that differs is patient identification. Even though our FLS required no additional staff, our model required an estimated $5 \mathrm{~min}$ of extra secretarial time per patient, both for identification and investigating if the patient was recently examined. Altogether, the estimated additional time spent by the secretaries to carry out these tasks amounted to approximately ten work days per year.

Since the effect of medication on fracture risk is undisputed, it seems unethical to design randomized studies for evaluation of FLS programs. Ganda et al. [12] summarised the evidence of the effectiveness of different FLS and graded them depending on the degree of involvement: if an osteoporosis unit both investigated and treated (type A), if an osteoporosis unit investigated but sent treatment recommendations to the primary care physician (type B), if the hospital informed the primary care physician of the fracture episode (type C) or if the hospital informed the patient of being at risk for a refracture and should become investigated (type D). Our FLS mostly resembled a type B FLS, but since a proportion of patients were treated by the hospital staff, the FLS to some extent resembled a type A service. Type B FLS models were able to increase DXA utilization from 9 to $59 \%$ [12]. Our minimal resource FLS increased the utilization rates from 8 to $40 \%$. However, the follow-up data of the late participants in the study were truncated at the end of the period. If only looking at 2011 participants versus those in 2013, the increase was larger, from 9 to $50 \%(p<0.001)$, thus almost as good as a typical type B model. When comparing the ability to get patients to treatment, our minimal effort FLS increased from $13 \%(2011-2012)$ to $32 \%(2013-2014)(p<0.001)$ or from $14 \%$ (2011) to $39 \%$ (2013) $(p<0.001)$. This is an increase well in line with the type B FLS increase from 20 to $41 \%$ $(p=0.01)$ and almost as efficient as a type A service, increasing treatment from 18 to $46 \%(p<0.001)[12]$.

In 2008, Dell et al. described the Kaiser Southern California Healthy Bones Program, a comprehensive program including 620,000 patients, and showed a $37 \%$ average reduction in hip fracture rate from 2006 versus an expected rate based on age-adjusted hip fracture rates from 1997 to 1999 [24]. Lih et al. [13] described a 4-year prospective study with 1544 patients in Sydney, Australia, and reported an $80 \%$ reduction of risk of further fracture. Controls, however, were chosen from the group that chose not to attend the FLS, which introduces potential selection bias issues. As opposed to Lih et al., we used historical controls. In Lund, Sweden, Åstrand et al. [14] reported a $42 \%$ risk reduction in a screened group in a 6-year follow-up of 592 fracture patients 50-75 years of age with a major osteoporotic fracture. However, a timedependent Cox model was not used and there were crossover effects from the control to the intervention group. Furthermore, a questionnaire was used to register re-fractures, with a response rate of $77 \%$, and self-reported fractures are known to be associated with uncertainty [18-20]. In the present study, we used a time-dependent Cox model, truncated the follow-up in order to avoid cross-over effects and used manually verified X-ray records for re-fracture reporting.

Huntjens et al. [16] in the Netherlands compared patients with non-vertebral fractures presenting at a hospital with FLS 
( $n=1412)$ with ditto patients at a neighbouring hospital without FLS $(n=1910)$. There was no difference in re-fracture rate after 2 years $(6.7$ versus $6.8 \%)$, but a time-dependent Cox model showed a $56 \%$ reduction of non-vertebral fractures and a $35 \%$ reduction of mortality, but the result could have been affected by the differences in population demographics between the two hospitals. Van Der Kallen et al. [15] compared, in a 2-year follow-up, 214 FLS patients with 220 patients who had chosen not to participate in the FLS, did not adjust for mortality and used a questionnaire to report rate of re-fracture and found a reduction of fracture rate from 16.4 to $5.1 \%$. Choosing non-participants as controls exposes the result for a potential selection bias.

In 2015, Nakayama et al. reported a $30 \%$ reduction in refracture rate over a 3-year follow-up period when comparing an FLS hospital with a non-FLS hospital [17]. However, the hospitals had different sizes and treated somewhat different patient groups, indicating possible problems of selection bias. Furthermore, the fact that the number of re-fractures was quite few suggests inadequate statistical power. Our study was performed in the same hospital with historical controls. Apart from Nakayama's study, the above studies [13-16,24] included patients actually participating in an FLS program whereas our study included all potential patients being eligible and compared data from the FLS period with a historic period, minimizing the risk of selection bias. In our study, $68 \%$ of the patients offered participation accepted a DXA examination and/or medication.

Lih et al. showed that the FLS association with re-fracture rate remained significant in the elderly when calculating separately for patients above and below 70 years of age [13]. Our study demonstrates that osteoporosis treatment was associated with equal or greater relative risk reduction in patients older than 80 years of age than in all treated patients in the study, 54 versus $51 \%$.

Thus, several previous studies [13-15, 17, 24] have reported that an FLS is associated with reduced fracture rates, but many studies suffer from different quality issues and lack power calculations. Using a post hoc power analysis on reported re-fracture rate in the control versus the intervention group, most studies [13-15, 17] appear to have sufficient power (73-100\%). However, even with high power, the results could be affected by quality issues such as uncertainty regarding self-reported re-fractures, differences in demographics between control and intervention hospitals or selection bias when using no-shows as control. Therefore, it could be of interest to reassess the post hoc power analysis, but instead use a prediction model for re-fracture rates in the FLS. Assuming that the re-fracture rates of the control group were true, the re-fracture rates of the intervention group can be calculated based on reported treatment rates (if not reported, $10 \%$ in the control group versus $50 \%$ in the FLS were assumed) and a $50 \%$ fracture risk reduction from treatment.
Using these assumptions, the statistical power of the previous studies would range from 7 to $54 \%$ [13-17], while our study reaches $51 \%$. Assuming a 4-month delay before the patient is likely to receive treatment after index fracture resulted in further reduced statistical power to between 7 and $39 \%$ for the previous studies and to $24 \%$ in our study. Persistence rates are not included in the model, which are known to be low, around $50 \%$ [25], which would decrease the difference in re-fracture rate and reduce the expected power even further. Thus, all yet existing studies investigating re-fracture reduction by FLS, including the present study, appear to be underpowered.

The present study investigating a minimal resource FLS, implemented using already existing staff, clearly demonstrates large effects in increasing DXA investigation and treatment rates. Among the strengths of our study is the fact that it covers a large patient group $(n=5329)$ with comprehensive background data on comorbidities and medications. The manual verification of the registers using patient charts and X-ray reports strengthens the quality of re-fracture data and offers a reliable re-fracture rate. Also, clinical vertebral fractures, often omitted in other studies, were included in our study. Furthermore, our control group is clearly defined with the same patient category from the same hospital and patient preference was not an inclusion factor.

\section{Conclusions}

We conclude that a minimal resource FLS increased the number of fracture patients being DXA-examined and treated, resembling increases observed in coordinator-based FLS models. Patients receiving osteoporosis medication had reduced risk of any major osteoporotic, vertebral and hip fracture. Furthermore, the studied FLS was related to reduced refracture rate in hip fracture patients 80 years or older, but this result could have been affected by insufficient statistical power in the analysis.

Acknowledgments The authors gratefully acknowledge the support from the research fund at Skaraborg Hospital, Skövde, Sweden, and in particular Salmir Nasic for statistical support. We also wish to thank Hulda Vigfusdottir, Anette Carlsson and John Karlsson Sondell for extensive data collection as well as all staff involved in the FLS.

Compliance with ethical standards The study was approved by the regional ethical review board in Gothenburg.

Conflicts of interest None.

Funding sources This study was funded by the research fund at Skaraborg Hospital Skövde, Sweden, the Swedish Research Council (VR), the Gothenburg Society of Medicine (GLS), the ALF/LUA grant from the Sahlgrenska University Hospital and Gustaf V:s och Drottning Victorias Frimurarstiftelse. 
Open Access This article is distributed under the terms of the Creative Commons Attribution-NonCommercial 4.0 International License (http:// creativecommons.org/licenses/by-nc/4.0/), which permits any noncommercial use, distribution, and reproduction in any medium, provided you give appropriate credit to the original author(s) and the source, provide a link to the Creative Commons license, and indicate if changes were made.

\section{References}

1. Murray CJ, Barber RM, Foreman KJ et al (2015) Global, regional, and national disability-adjusted life years (DALYs) for 306 diseases and injuries and healthy life expectancy (HALE) for 188 countries, 1990-2013: quantifying the epidemiological transition. Lancet (Lond, Engl). doi:10.1016/s0140-6736(15)61340-x

2. Borgstrom F, Zethraeus N, Johnell O, Lidgren L, Ponzer S, Svensson O, Abdon P, Ornstein E, Lunsjo K, Thorngren KG, Sernbo I, Rehnberg C, Jonsson B (2006) Costs and quality of life associated with osteoporosis-related fractures in Sweden. Osteoporos Int 17(5):637-650. doi:10.1007/s00198-005-0015-8

3. Sambrook P, Cooper C (2006) Osteoporosis. Lancet 367(9527): 2010-2018. doi:10.1016/s0140-6736(06)68891-0

4. Sattui SE, Saag KG (2014) Fracture mortality: associations with epidemiology and osteoporosis treatment. Nat Rev Endocrinol 10(10):592-602. doi:10.1038/nrendo.2014.125

5. Lorentzon M, Cummings SR (2015) Osteoporosis: the evolution of a diagnosis. J Intern Med 277(6):650-661. doi:10.1111/joim.12369

6. Wells George A, Cranney A, Peterson J, Boucher M, Shea B, Welch V, Coyle D, Tugwell P (2008) Alendronate for the primary and secondary prevention of osteoporotic fractures in postmenopausal women. Cochrane Database Syst Rev (1). doi:10.1002/14651858. CD001155.pub2

7. Black DM, Delmas PD, Eastell R, Reid IR, Boonen S, Cauley JA, Cosman F, Lakatos P, Leung PC, Man Z, Mautalen C, Mesenbrink $\mathrm{P}, \mathrm{Hu} \mathrm{H}$, Caminis J, Tong K, Rosario-Jansen T, Krasnow J, Hue TF, Sellmeyer D, Eriksen EF, Cummings SR (2007) Once-yearly zoledronic acid for treatment of postmenopausal osteoporosis. $\mathrm{N}$ Engl $\mathrm{J}$ Med 356(18):1809-1822. doi:10.1056/NEJMoa067312

8. Boonen S, Adachi JD, Man Z, Cummings SR, Lippuner K, Torring O, Gallagher JC, Farrerons J, Wang A, Franchimont N, San Martin J, Grauer A, McClung M (2011) Treatment with denosumab reduces the incidence of new vertebral and hip fractures in postmenopausal women at high risk. J Clin Endocrinol Metab 96(6):17271736. doi:10.1210/jc.2010-2784

9. Klotzbuecher CM, Ross PD, Landsman PB, Abbott TA, Berger M (2000) Patients with prior fractures have an increased risk of future fractures: a summary of the literature and statistical synthesis. J Bone Miner Res 15(4):721-739. doi:10.1359/jbmr.2000.15.4.721

10. Quality and Efficiency in Swedish Health Care - Regional Comparisons 2013 by the Swedish Association of Local Authorities and Regions (2013)

11. Sale JE, Beaton D, Posen J, Elliot-Gibson V, Bogoch E (2011) Systematic review on interventions to improve osteoporosis investigation and treatment in fragility fracture patients. Osteoporos Int 22(7):2067-2082. doi:10.1007/s00198-011-1544-y

12. Ganda K, Puech M, Chen JS, Speerin R, Bleasel J, Center JR, Eisman JA, March L, Seibel MJ (2013) Models of care for the secondary prevention of osteoporotic fractures: a systematic review and meta-analysis. Osteoporos Int 24(2):393-406. doi:10.1007/ s00198-012-2090-y

13. Lih A, Nandapalan H, Kim M, Yap C, Lee P, Ganda K, Seibel MJ (2011) Targeted intervention reduces refracture rates in patients with incident non-vertebral osteoporotic fractures: a 4-year prospective controlled study. Osteoporos Int 22(3):849-858. doi:10.1007/ s00198-010-1477-x

14. Astrand J, Nilsson J, Thorngren KG (2012) Screening for osteoporosis reduced new fracture incidence by almost half: a 6-year follow-up of 592 fracture patients from an osteoporosis screening program. Acta Orthop 83(6):661-665. doi:10.3109/17453674.2012. 747922

15. Van der Kallen J, Giles M, Cooper K, Gill K, Parker V, Tembo A, Major G, Ross L, Carter J (2014) A fracture prevention service reduces further fractures two years after incident minimal trauma fracture. Int J Rheum Dis 17(2):195-203. doi:10.1111/1756-185X. 12101

16. Huntjens KM, van Geel TA, van den Bergh JP, van Helden S, Willems P, Winkens B, Eisman JA, Geusens PP, Brink PR (2014) Fracture liaison service: impact on subsequent nonvertebral fracture incidence and mortality. J Bone Joint Surg Am 96(4):e29. doi:10. 2106/JBJS.L.00223

17. Nakayama A, Major G, Holliday E, Attia J, Bogduk N (2015) Evidence of effectiveness of a fracture liaison service to reduce the re-fracture rate. Osteoporos Int. doi:10.1007/s00198-0153443-0

18. Chen Z, Kooperberg C, Pettinger MB, Bassford T, Cauley JA, LaCroix AZ, Lewis CE, Kipersztok S, Borne C, Jackson RD (2004) Validity of self-report for fractures among a multiethnic cohort of postmenopausal women: results from the Women $\mathrm{s}$ Health Initiative observational study and clinical trials. Menopause 11(3):264-274. doi:10.1097/01.gme.0000094210. 15096.fd

19. Ismail AA, O'Neill TW, Cockerill W, Finn JD, Cannata JB, Hoszowski K, Johnell O, Matthis C, Raspe H, Raspe A, Reeve J, Silman AJ (2000) Validity of self-report of fractures: results from a prospective study in men and women across Europe. EPOS Study Group. European Prospective Osteoporosis Study Group. Osteoporos Int 11(3):248-254

20. Nevitt MC, Cummings SR, Browner WS, Seeley DG, Cauley JA, Vogt TM, Black DM (1992) The accuracy of self-report of fractures in elderly women: evidence from a prospective study. Am J Epidemiol 135(5):490-499

21. Marsh D, Akesson K, Beaton DE, Bogoch ER, Boonen S, Brandi ML, McLellan AR, Mitchell PJ, Sale JE, Wahl DA (2011) Coordinator-based systems for secondary prevention in fragility fracture patients. Osteoporos Int 22(7):2051-2065. doi:10.1007/ s00198-011-1642-x

22. The National guidelines for Musculoskeletal Diseases by The National Board of Health and Welfare (2012)

23. Bolland MJ, Grey AB, Gamble GD, Reid IR (2010) Effect of osteoporosis treatment on mortality: a meta-analysis. J Clin Endocrinol Metab 95(3):1174-1181. doi:10.1210/jc.2009-0852

24. Dell R, Greene D, Schelkun SR, Williams K (2008) Osteoporosis disease management: the role of the orthopaedic surgeon. J Bone Joint Surg Am 90(Suppl 4):188-194. doi:10.2106/JBJS.H.00628

25. Kothawala P, Badamgarav E, Ryu S, Miller RM, Halbert RJ (2007) Systematic review and meta-analysis of real-world adherence to drug therapy for osteoporosis. Mayo Clin Proc 82(12):14931501. doi:10.1016/s0025-6196(11)61093-8 